\title{
Neoplasia Trofoblástica Gestacional en H.U.V. Cali - Colombia Enero 1o. de 1979 - Diciembre 31 de 1983
}

\author{
Dres.: J. E. Zúñiga, Gilberto Mesa (R3), y Freddy Briceño (DA)
}

\section{INTRODUCCION}

Las neoplasias trofoblásticas gestacionales se originan de trofoblasto del embarazo humano. Ellas son injertos de tejido maligno coriónico fetal en el huésped materno. Producen en forma invariable hCG, la cual está directamente relacionada con el número de células tumorales vivas presentes. Desde la introducción de la Quimioterapia se han convertido en una de las neoplasias de mayor curabilidad.

Estas neoplasias incluyen: Mola hidatidiforme. Mola invasiva (coriadenoma destrens) y coriocarcinoma (corioepitelioma).

En la mola prolifera trofoblasto de revestimiento de las vellocidades con edema y agrandamiento de las mismas y desaparición o extrema rareza de vasos sanguíneos.

Herting sugiere que hay agenesia o degeneración temprana del sistema cardiovascular embrionario (3-5 semanas) en presencia de circulación materna conti- nuada con trofoblasto funcionante, resultando, edema del tejido velloso.

Pask sugiere lo contrario; trofoblasto anormal, edematoso lleva a muerte del embrión.

La mayoría de las molas son $x x$ tretraploides; el coriocarcinoma no parece tener relación con cromatina.

El coriocarcinoma es muy maligno, puede seguir a un parto normal, aborto o mola, aunque a veces no se detecta evento obstétrico previo. Suele manifestarse como masa obscura, hemorrágica, grumosa, en pared uterina, a veces intramural, que puede causar perforación y hemoperitoneo, suele haber proliferación desordenada de tejido trofoblástico del músculo, al cual destruye causando hemorragia, necrosis de coagulación y perdida del patrón de vellosidad.

Debido a la importancia de estas neoplasias se hizo una revisión retrospectiva de los casos diagnósticos en el H. U. V.. 
Cali, Colombia entre 1o. de enero/79 a diciembre 31/83. Haciendo énfasis en los casos de coriocarcinoma y haciendo comparación de la incidencia de estas neoplasias en nuestro Hospital, con estudios en otros sitios del país y en este mismo centro entre 1962/1972.

\section{PACIENTES Y METODOS}

Se analizaron 69 casos de neoplasia trofoblástica gestacional diagnosticados en $H$. U. $V$. en el período entre 10. enero/79 a diciembre 31/83. En este lapso de tiempo se atendieron en el $\mathrm{H}$. U. V. 43.547 pacientes obstétricas presentándose 29.665 partos, 9.960 cesáreas, 3.922 embarazos que terminaron a las 20 semanas (Ectópicos y abortos).

INCIDENCIA 1 Mola x 631 embarazos.

$$
1 \text { Mola } x 574 \text { partos. }
$$

Ocurrieron 15 casos de Coriocarcinoma: con una incidencia:

\section{1: 2.903 embarazos}

1: 2.641 partos

Se efectuó un estudio retrospectivo de las historias clínicas de pacientes con diagnóstico confirmado por anatomía patológica de mola hidatidiforme, corioadenoma destrues, o mola invasiva y coriocarcinoma, pacientes tratadas en salas generales y la consulta externa del servicio de Gineco-Obstetricia del H. U. V. (Hospital Universitario del Valle).

Para cada caso se estableció un protocolo que incluia: procedencia, área, grupo etario, paridad, sintomatología, hallazgos clínicos, estudios paraclínicos, antecedentes obstétricos previo a la aparición del cuadro, sitios de metastasis, seguimiento con HCG, tratamiento y remisión. Para la anatomía patológica se siguió la clasificación:
A. Mola con leve actividad trofoblástica.
B. Mola con moderada actividad trofo- blástica.
C. Mola con severa actividad trofoblás- tica.
D. Cariodenoma.
E. Coriocarcinoma.

Finalmente se analizan los mismos parámetros para cada uno de los 15 casos de diagnóstico de coriocarcinoma.

\section{RESULTADOS}

CUADRO No. 1

DISTRIBUCION CASOS DIAGNOSTICADOS X GRUPO ETARIO

\begin{tabular}{|l|c|c|c|c|c|c|}
\hline Grupo Etario & $15 \mathrm{~A}$ & $15-20$ & $21-30$ & $31-40$ & $40 \mathrm{~A}$ & Sin datos \\
\hline No Casos & 2 & 21 & 28 & 7 & 6 & 5 \\
\hline Porcentaje & 2.88 & 30.48 & 40.58 & 108 & 8.68 & 7.28 \\
\hline
\end{tabular}

Se presentaron en este período $69 \mathrm{ca}$ sos de neoplasia gestacional trofoblástica, 48 casos (69\%) molas hidatidiformes, 15 coriocarcinomas (21.1응 y 7 casos de corioadenomas (10\%).

42 pacientes (60.8\%) procedían de Cali y 24 (34.8\%) venían de otras regiones del Valle o del País. En 3 casos no se consigno la procedencia. 41 casos (60\%) vivían en zona urbana y el resto eran de zona rural. De acuerdo con la distribución por grupos de edad sólo se encontraron 2 casos (2.8응 en menores de 15 años y 6 casos $(8.6 \%$ ) en mujeres de más de 40 años. 
La mayor incidencia ocurrió entre 15 y 30 años: 49 casos para $71 \%$.

\section{Molas Hidatidiformes 48 casos $69 \%$ \\ Corioadenomas $\quad 7 \operatorname{casos} 10 \%$ \\ Coriocarcinomas $\quad 15 \operatorname{casos} 21.1 \%$}

CUADRO No. 2

DISTRIBUCION DE CASOS SEGUN PARIDAD

\begin{tabular}{|ccc|}
\hline Paridad & No. Casos & Porcentaje \\
\hline 0 & 26 & 37.6 \\
\hline 1 & 18 & 26.1 \\
\hline 2 & 9 & 13 \\
\hline 3 & 7 & 10.1 \\
\hline 4 o más & 6 & 8.6 \\
\hline Sin dato & 3 & 4.0 \\
\hline
\end{tabular}

El mayor número de casos se presentó en pacientes sin embarazos previos 26 (37.6\%) seguido de las pacientes que tenían 1 solo hijo: 18 (26.1\%) y en las de 4 o más hijos ocurrieron 6 casos (8.6\%).

\section{CUADRO No. 3}

\section{SINTOMAS Y SIGNOS CLINICOS PREDOMINANTES}

\begin{tabular}{|l|c|c|}
\hline \multicolumn{1}{|c|}{ Sintomatología } & No. & 8 \\
\hline Sangrado genital & 57 & 82.6 \\
\hline $\begin{array}{l}\text { Expulsión de vesículas } \\
\text { por vagina }\end{array}$ & 17 & 24.6 \\
\hline $\begin{array}{l}\text { Síntomas de toxemia } \\
\text { temprana }\end{array}$ & 9 & 13.04 \\
\hline Amenorrea & 22 & 31.8 \\
\hline $\begin{array}{l}\text { Síntomas simpáticos de } \\
\text { embarazo exacerbados }\end{array}$ & 22 & 31.8 \\
\hline Síntomas generales & 2 & 2.8 \\
\hline Tos, hemoptisis, disnea & 1 & 1.4 \\
\hline
\end{tabular}

SINTOMAS Y SIGNOS CLINICOS PREDOMINANTES

\begin{tabular}{|lcr|}
\hline Examen físico & No. & \multicolumn{1}{c|}{$\%$} \\
\hline $\begin{array}{l}\text { Discordancia entre tamaño } \\
\text { uterino y amenorrea }\end{array}$ & 43 & 62.3 \\
\hline No se palpan partes fetales & 34 & 49.2 \\
\hline Quistes teca-luteínicos & 15 & 21.7 \\
\hline Toxemia temprana & 6 & 8.6 \\
\hline Masa en vagina & 3 & 4.3 \\
\hline
\end{tabular}

El sangrado vaginal ocurrió en $82.6 \%$ de las pacientes: 57 casos. La amenorrea se presentó en $31.8 \% 22$ casos. $Y$ en la exacerbación de los síntomas simpáticos del embarazo fue manifestación en 22 casos: $31.8 \%$ cuadro No. 3. Respecto a los hallazgos clínicos la discordancia entre altura uterina y amenorrea en favor de la primera se detectó en 43 casos: $62.3 \%$ la imposibilidad para palpar partes fetales $y$ la ausencia de fetocardia en úteros aumentados de tamaño fueron hallazgos presentes en 34 casos (49.2응).

Llama la atención la alta incidencia de masas parauterinas 15 casos: $21.7 \%$

CUADRO No. 4

\section{ANTECEDENTE OBSTETRICO RECIENTE PREVIO AL DIAGNOSTICO CLINICO}

\begin{tabular}{|lcc|}
\hline Antecedentes obstétricos & No. & $\%$ \\
\hline $\begin{array}{l}\text { Embarazo normal parto } \\
\text { cesárea }\end{array}$ & 15 & 21.7 \\
\hline Mola hidatidiforme & 13 & 18.8 \\
\hline Aborto & 6 & 8.6 \\
\hline Embarazo ectópico & 1 & 1.4 \\
\hline Total & 35 & $50 \%$ \\
\hline
\end{tabular}


En 26 casos (casi 38\%) no había antecedente obstétrico y en 15 casos $(21.7 \%)$, ; tenían antecedentes de embarazo normal terminado por parto normal o cesárea.

En 38 casos (55\%) las HCG mostraron títulos muy elevados y fue la ecografía el estudio paraclínico que aparte del anterior sirvió más frecuentemente para establecer el diagnóstico en 22 casos (31.8\%). Cuadro No. 5.

CUADRO No. 5

ESTUDIOS PARACLINICOS PARA CONFIRMAR EL DIAGNOSTICO

\begin{tabular}{|llcc|}
\hline Laboratorio & No. & \% \\
\hline $\begin{array}{c}\text { Gonadotropina } \\
>\end{array}$ & $\begin{array}{l}100.000 \mathrm{us} \\
\text { orina } 24 \mathrm{hrs} \\
40.000 \mathrm{u} / \mathrm{ml}\end{array}$ & 38 & 55 \\
\hline $\begin{array}{l}\text { Rx abdomen } \\
\text { Ausencia partes fetales }\end{array}$ & 5 & 7.2 \\
\hline $\begin{array}{l}\text { Gonadotropina } \\
<\end{array}$ & $\begin{array}{l}100.000 \mathrm{us} \\
\text { orina } 24 \mathrm{hrs} \\
40.000\end{array}$ & 16 & 23.1 \\
\hline Ecografia & & 22 & 31.8 \\
\hline
\end{tabular}

La anatomía patológica evidenció alta incidencia de molas con leve y moderada actividad trofoblástica: 44 casos (63.6\%) Coriocarcinoma 15 casos (21.7을 sólo 3 casos se internaron como molas de severa actividad trofoblástica.

CUADRO No. 6

SITIO DE LA METASTASIS

\begin{tabular}{|lcc|}
\hline Organo & No. & $\%$ \\
\hline Pulmón & 14 & 20.3 \\
\hline Vagina & 6 & 8.6 \\
\hline Hígado & 1 & 1.4 \\
\hline Cerebro & 1 & 1.4 \\
\hline Total & 22 & $32 \%$ \\
\hline
\end{tabular}

Al revisar la presencia de metastasis el pulmón fue el órgano más frecuentemente atacado: 14 casos (20.3응 seguido de la vagina en 6 casos (8.6응 cerebro y el hígado sólo fueron atacados en 1 caso cada uno, y 47 casos (68\%) no hubo evidencia de metastasis.

CUADRO No. 7

\begin{tabular}{|lcr|}
\hline Tratamiento & No. & \multicolumn{1}{c|}{ ○ } \\
\hline Dilatación y legrado & 39 & 56.5 \\
\hline Histerectomía & 10 & 14.5 \\
\hline Quimioterapia - MAC & 6 & 8.6 \\
\hline $\begin{array}{l}\text { Quimioterapia + } \\
\text { Histerectomía }\end{array}$ & 10 & 14.5 \\
\hline Radioterapia & 1 & 1.4 \\
\hline
\end{tabular}

\section{METODO DE TRATAMIENTO $Y$ SEGUIMIENTO CON HCG}

El tratamiento más usado fue la evacuación con dilatación y legrado 39 ca$\operatorname{sos}(56 \%)$ la histerectomia sola (10 casos) o acompañada de Quimioterapia (10 casos) fue el segundo método empleado $29 \%$. La Quimioterapia sólo se usó en 6 casos (8.6\%).

\section{CUADRO No. 8}

\begin{tabular}{|lcc|}
\hline $\begin{array}{l}\text { Seguimiento con } \\
\text { gonadotropinas }\end{array}$ & No. & $\%$ \\
\hline $\begin{array}{l}\text { Negativas Post-evacuación } \\
\text { o histerectomia }\end{array}$ & 45 & 65.2 \\
\hline Positiva después de 60 días & 3 & 4.3 \\
\hline Corioadenoma & 7 & 10.1 \\
\hline Coriocarcinoma & 15 & 21.7 \\
\hline
\end{tabular}


CUADRO No. 9

\begin{tabular}{|lcc|}
\hline Remisión de la enfermedad & Casos & \multicolumn{2}{l|}{ 을 } \\
\hline Negativo con 1 ciclo de Quimioterapia & 2 & 2.8 \\
\hline Negativo con 3 ciclos de Quimioterapia & 8 & 11.6 \\
\hline $\begin{array}{l}\text { Negativo con Dilatación y curetaje o } \\
\text { Histerectom ia solos }\end{array}$ & 45 & 65.2 \\
\hline Negativo con MAC Histerectomia & 6 & 8.6 \\
\hline Muerte con MAC Histerectomia & 1 & 1.4 \\
\hline Muerte con MAC & 1 & 1.4 \\
\hline No regresaron a control & 6 & 8.6 \\
\hline
\end{tabular}

\section{DISTRIBUCION DE LOS CASOS} SEGUN AREA DE PROCEDENCIA EDAD Y PARIDAD. 15 CASOS CORIOCARCINOMA H.U.V. $1979-1983$

En relación con los casos de enfermedades trofoblásticas maligna coriocarcinoma se diagnosticaron en ese período 15 casos. La mayoría 7 casos (46.6응 procedían de Cali y vivían en áreas urbanas: 10 casos (66\%) y según la paridad hubo poca correlación con la misma, ya que ocurrieron 3 casos en nulíparas, 3 en mujeres con 1 y 2 hijos respectivamente.

Procedentes de Cali 7 casos (46.6\%)

Del Area urbana $10 \operatorname{casos}$ (66.9응

Se presentaron 3 casos en nulíparas

CUADRO No. 10

CORIOCARCINOMA PROCEDENCIA URBANA O RURAL

\begin{tabular}{|lcc|}
\hline Area & No. & $\frac{\circ}{0}$ \\
\hline Urbana & 10 & 66.6 \\
\hline Rural & 5 & 33.3 \\
\hline
\end{tabular}

CORIOCARCINOMA DISTRIBUCION POR EDAD

\begin{tabular}{|lll|}
\hline Edad & No. & \% \\
\hline Menor de 15 años & 0 & - \\
\hline $15-20$ años & 4 & 26.6 \\
\hline $21-30$ años & 5 & 33.3 \\
\hline $31-40$ años & 2 & 13.3 \\
\hline Mayor de 40 años & 2 & 13.3 \\
\hline Sin dato & 2 & 13.3 \\
\hline
\end{tabular}

CORIOCARCINOMA SEGUN PARIDAD

\begin{tabular}{|lll|}
\hline Paridad & No. & $\frac{\circ}{0}$ \\
\hline 0 & 3 & 20 \\
\hline 1 & 3 & 20 \\
\hline 2 & 3 & 20 \\
\hline 3 & 2 & 13.3 \\
\hline 4 o más & 4 & 26.6 \\
\hline
\end{tabular}

\section{CORIOCARCINOMA}

\begin{tabular}{|lcc|}
\hline Síntomas y signos & No. & $\%$ \\
\hline Sangrado vaginal & 13 & 86.6 \\
\hline Amenorrea & 3 & 20 \\
\hline Expulsión vesicular & 3 & 20 \\
\hline Síntomas generales & 1 & 5.6 \\
\hline Quistes Ováricos & 5 & 33.3 \\
\hline $\begin{array}{l}\text { Discordancia y Amenorrea } \\
\text { tamaño uterino }\end{array}$ & 3 & 20 \\
\hline Tumor vaginal & 3 & 20 \\
\hline Tumor cervix uterino & 1 & 6.6 \\
\hline
\end{tabular}


El sangrado vaginal al igual que en la mola fue el hallazgo predominante en la sintomatología de los casos de coriocarcinoma. 13 casos (86.6\%). Siendo la presencia de quistes tecalutéinicos 5 casos (33.3\%), la presencia de masa vaginal 3 casos (20\%) y las discordancia Au/Am 3 casos (20\%) los hallazgos más frecuentes al examen físico.

Es importante ver que la $R x$ de torax fue $(+)$ para metastasis en 7 casos (46.6응.

CUADRO No. 11

HALLAZGOS CLINICOS

$Y$ DE LABORATORIO EN 15 CASOS DE CORIOCARCINOMA H.U.V. 1979 - 1983

\begin{tabular}{|cccc|}
\hline Laboratorio coriocarcinoma & No. & $\%$ \\
\hline $\begin{array}{c}\text { Gonodotropina } \\
>\end{array}$ & $\begin{array}{c}100.000 \text { us orina } \\
40.000 \mathrm{us} / \mathrm{ml}\end{array}$ & 5 & 33 \\
\hline $\begin{array}{c}\text { Gonodotropina } \\
<\end{array}$ & $\begin{array}{c}100.000 \mathrm{us} \text { orina } \\
40.000 \mathrm{us} / \mathrm{ml}\end{array}$ & 5 & 33 \\
\hline Rx Torax $(+)$ metastasis & 7 & 46.6 \\
\hline
\end{tabular}

\section{ANTECEDENTE OBSTETRICO EN CORIOCARCINOMA 15 CASOS HUV 1979 - 1983}

\begin{tabular}{|lr|}
\hline Mola Hidatidiforme & $6 \operatorname{casos}(40 \%)$ \\
\hline Parto normal o cesárea & $4 \operatorname{casos}(26.6 \%)$ \\
\hline No de tectado & $4 \operatorname{casos}(26.6 \%)$ \\
\hline Aborto & 1 caso (6.6\%) \\
\hline Total & $15 \operatorname{casos}(100 \%)$ \\
\hline
\end{tabular}

1 caso se presentó después de parto normal seguido de 7 meses de sangradio vaginal persistente.
1 caso Dx. mola a los 2 meses prueba de Galli (-), al cabo de 2 años regresa y se le hace Dx. coriocarcinoma.

2 casos antecedente de cesáera.

1 caso: 1 año de sangrado vaginal sometida a 7 legrados sin hacer Dx. correcto.

METASTASIS DE CORIOCARCINOMA 15 CASOS HUV. 1979 - 1983

\begin{tabular}{|lll|}
\hline Organo & No. & $\div$ \\
\hline Pulmón & 12 & 80 \\
\hline Vagina & 6 & 40 \\
\hline Hígado & 1 & 6.6 \\
\hline Cerebro & 1 & 6.6 \\
\hline
\end{tabular}

Se nota claramente como el pulmón es el sitio predilecto para la metástasis de este tumor seguido de la vagina. El número de casos y los porcentajes varían porque en un mismo paciente se dan varios sitios metastásicos.

En todos los casos se uso la Quimioterapia; como droga de elección siempre se consideró el MTX. Dependiendo del caso, se empleo el esquema clásico MAC.

\section{TRATAMIENTO DE CORIOCARCINOMA EN 15 CASOS EN H.U.V.}

\begin{tabular}{|lll|}
\hline Método de tratamiento & No. casos & $\%$ \\
\hline $\begin{array}{l}\text { Quimioterapia con } \\
\text { histerectomia }\end{array}$ & 9 & 60.0 \\
\hline Quimioterapia & 5 & 13.3 \\
\hline $\begin{array}{l}\text { Quimioterapia con } \\
\text { Histerectomia } \\
\text { radioterapia }\end{array}$ & 1 & 6.6 \\
\hline
\end{tabular}


Se puede notar la remisión con control de la enfermedad en el $86.6 \%$ de los casos con muertes en el $13.2 \%$ de los casos teniendo en cuenta que se trata en la mayoría de los casos de enfermedad Maligna Metastásica.

\section{REMISION DE LA ENFERMEDAD}

\begin{tabular}{|ll|}
\hline $\begin{array}{l}\text { Negativo con Histerectomia }+3 \\
\text { ciclos de MTX solo ó MAC }\end{array}$ & 9 (60\%) \\
\hline Negativo con MAC + Histerectomia & 3 (20\%) \\
\hline Negativo con Histerectomia sola & $1(6.6 \%)$ \\
\hline Muerte con MAC. + Histerectomia & $1(6.6 \%)$ \\
\hline Muerte con MAC & $1(6.6 \%)$ \\
\hline
\end{tabular}

\section{CONCLUSIONES}

Se presenta un estudio de 69 casos de enfermedad del Trofoblasto en el H.U.V. del Valle en Cali con una incidencia de 1 caso por 631 embarazos y 1 caso por 574 partos, en el período 1979 - 1983.

En este grupo se presentaron $15 \mathrm{ca}$ riocarcinomas metastásicos con una incidencia del $1 \times 2.903$ embarazos y $1 \times 2.641$ partos.

En sólo 2 casos se presentó la enfermedad antes de los 15 años. En 6 casos por encima de los 40 años.

En el $60 \%$ las pacientes provenían del área urbana de Cali.

La distribución según el estudio histológico fue:

\section{BIBLIOGRAFIA}

1. Organización Mundial de la Salud serie de informes técnicos 692, Ginebra 1983.

$\begin{array}{lr}\text { Mola } \mathrm{H}, & 48 \text { casos } \\ \text { Corioadenomas } & 7 \text { casos } \\ \text { Coriocarcinomas } & 15 \text { casos }\end{array}$

En el 38\% las mujeres eran nulíparas. $Y$ en el $26 \%$ sólo tenían 1 hijo.

El síntoma de mayor ocurrencia fue el sangrado genital en el $82.6 \%$

El hallazgo más frecuente al examen físico fue la discordancia entre el tamaño uterino y la amenorrea.

El $21 \%$ de los casos se asoció la enfermedad con masas para-uterinas, Quistes teca-luteínicos.

Los títulos aumentados de gonadotrofina coriónica junto con el estudio ecográfico fueron los examenes paraclínicos más útiles para el diagnóstico.

El pulmón y la vagina fueron los sitios preferidos para la metástasis.

Se logró controlar la enfermedad maligna coriocarcinoma metastásico en el $86 \%$ de los casos con un $13.2 \%$ de mortalidad. Este porcentaje de recuperación de pacientes nos parece bastante bueno en nuestro medio, con tantas limitaciones para estudio de los casos y para la administración de drogas citotóxicas sin el recurso del ácido folínico, para reducir el fenómeno de la toxicidad.

2. SURWIT E.A. Y HAMMOND CH. B. Neoplasias trofoblásticas de la gestación year Book de Obstetricia y ginecología 302-321. 1978. 
3. HAMMOND C.B.; BORCHERT, L. TYREY. L. et all. Treatment of metastatic trophoblastic Disease good and poor prognosis. Am. J. Obstet. Gynecol. 115: 4511973.

4. DISAIA. PH. J; CREASMAN, W.T. Clinical Gynecologic Oncology The C.V. Mosby Company 1981.

5. BERKOWITZ R.S.; GOLDSTEIN D.P.; et - all. Anticonceptivos orales $y$ enfermedad trofoblástica postmolar. Obstet Gynecol. 58: 474-477 octubre 1981.
6. SCHLAERTH J.B.; MORROW P; et all. Características pronósticas de la regresión del nivel sérico de gonadotrofina coriónica humana luego de un embarazo molar. Obstet. Gynecol. 58: 478-482 octubre, 1981.

7. WEHMANN R.E.; AYALA A.R. BIRKEN et all. Mejor supervisión de la neoplasia trofoblástica gestacional mediante la utilización de un estudio altamente sensitivo de la gonadotrofina coriónica humana urinaria. Am. J. Obstet. gynecol. 140: 753-757. Agosto 1, 1981. 